\title{
A Set of Time Series Prediction Models Based on Difference Method
}

\author{
Xiaoli Lu, Hongxu Wang*, Chengguo Yin and Hao Feng \\ Hainan Tropical Ocean University, Sanya, Hainan, China, 572022 \\ ${ }^{*}$ Corresponding author
}

\begin{abstract}
This paper proposed a set of time series prediction models based on difference method(ASD). For a time series, the computer can automatically find the time series search method to filter out the ideal in ASD prediction model. For example, the forecast number of registered at the University of Alabama in 1971 1992 years, the ideal forecasting model is $A_{j}$ $(0.000003,0.000003)$, which can make the mean square error $M S E=0$ and the average prediction error rate $A F E R=0 \%$, that thoroughly solve the unsatisfactory prediction accuracy of the existing fuzzy time series forecasting model.
\end{abstract}

Keywords-differential rate; a set of time series prediction models; ASD's sum of fraction functions $K_{j}(U, V)$

\section{INTRODUCTION}

In year 1993, Song and Chissom[1-4] firstly proposed fuzzy time series prediction model and successfully predicted the number of registered at the University of Alabama in year $1971 \sim 1992$, based on the fuzzy set theory, but the prediction accuracy is not satisfactory. The registered number is shown in table $\mathrm{C}$, and its distribution map is shown in figure 1 , that is actually a time series, which is undulating change radically. method to filter out the ideal in ASD prediction model $\mathrm{Aj}$ (0.000003,0.000003), mean square error $\mathrm{MSE}=0$ and the average prediction error rate of $\mathrm{AFER}=0 \%$, which obtain satisfactory prediction accuracy.

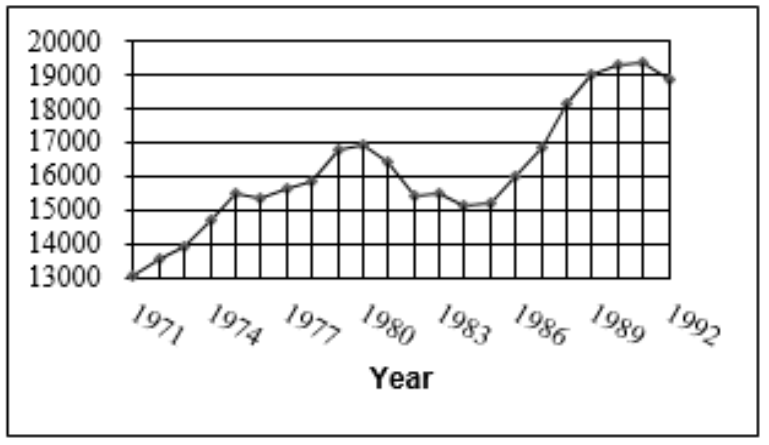

FIGURE I.DISTRIBUTION OF ENROLLMENT NUMBERS OF ALABAMA UNIVERSITY IN 1971 1992

Since then, the fuzzy time series forecasting model are emerging[6-15]. In year 2007, Jilani \& Burney \& Ardil ${ }^{[4,5]}$ used the concept of de-fuzzification, proposed a fuzzy time series forecasting model, simulated and predicted of the number of registered at the University of Alabama, obtained $\mathrm{AFER}=1.0242 \% \mathrm{MSE}=41426$ [5], which forecasting accuracy is the best since 2007, but still unsatisfactory. Affected by this literature, this paper proposed a set of time series prediction models based on ASD's sum of fraction functions $K_{j}(U, V)$, inverse function $H_{j}(U, V)$ and prediction function $A_{j}(U, V)$. This paper also proposed automatic search method and standard time series forecasting model concept. For the registered number of the University of Alabama in year 1971 1992, application of automatic time series search

\section{A Set of Time Series Prediction Models}

Define1: historical data sets $\mathrm{W}=\left\{\mathrm{W}_{1}, \mathrm{~W}_{2}, \ldots, \mathrm{W}_{\mathrm{n}}\right\}$. The difference of historical data is calculated as $\mathrm{Z}_{\mathrm{j}}=\mathrm{W}_{\mathrm{j}}-\mathrm{W}_{\mathrm{j}-1}$, and the difference field of historical data is $Z=\left\{Z_{2}, Z_{3}, \ldots, Z_{n}\right\}$.

$$
\operatorname{MSE}\left(\text { Mean Square Error): } S M E=\frac{1}{n-1} \sum_{j=2}^{n}\left(W_{j}-A_{j}\right)^{2}\right.
$$

AFER (Average Forecasting Error Rate:

$$
A F E R=\left(\frac{1}{n-1} \sum_{j=2}^{n}\left|W_{j}-A_{j}\right| / W_{j}\right) \times 100 \%,
$$

$A_{j}$ is the prediction number in year $\mathrm{j}$.

Define2: For $\mathrm{j} \in\{2,3, \ldots, \mathrm{n}\}$, and define that $(\mathrm{u} \in(0,1))$ :

$$
\begin{aligned}
& K_{j}(u, v)=\left\{\begin{array}{l}
\frac{1}{Z_{2}}+\frac{v}{Z_{3}}, j=2, \\
\frac{u}{Z_{j-1}}+\frac{1}{Z_{j}}, j \in\{3,4, \cdots, n\} .
\end{array} H_{j}(u, v)=\left\{\begin{array}{l}
\frac{1+v}{\frac{1}{Z_{2}}+\frac{v}{Z_{3}}}, j=2, \\
\frac{u+1}{\frac{u}{Z_{j-1}}+\frac{1}{Z_{j}}}, j \in\{3,4, \cdots, n\} .
\end{array}\right.\right. \\
& A_{j}(u, v)=W_{j-1}+H_{j}(u, v) .
\end{aligned}
$$

If $\mathrm{u}$ and $\mathrm{V}$ are respectively independent variables on specific values within the domain, established a prediction formula of $\mathrm{Aj}(\mathrm{U}, \mathrm{V})$. which can simulate the historical data to predict the time series.

Define3: If $\mathrm{u}$ and $\mathrm{V}$ are respectively independent variables on specific values within the domain, many prediction model $\mathrm{A}_{\mathrm{j}}(\mathrm{U}, \mathrm{V})$ can be obtained, that is a set of time series prediction models based on difference method, abbreviated as ASD.

The general element of ASD is $A_{j}(u, v)$. $A_{j}(u, v)$ not only represents the prediction formula, but also prediction models. 
Define4: If MSE $=0$ and AFER $=0 \%$, which called that the prediction model $\mathrm{A}_{\mathrm{j}}(\mathrm{u}, \mathrm{v})$ is ideal.

Define5: For a time series, application of computer automatically find the time series standard in ASD prediction model, this method is called automatic searching method. The main steps are: for a time sequence from a decimal as the starting point, programming, search, calculation,... Until the ideal time series prediction model $\mathrm{A}_{\mathrm{j}}(\mathrm{U}, \mathrm{V})$ is selected out of ASD $(\mathrm{AFER}=0 \%$, MSE $=0$ )

Example1: Predict registration number of Alabama University in year 1971 1992, from the starting point $\mathrm{u}=\mathrm{v}=0.0003$, select $\mathrm{u}=\mathrm{v}=0.0003, \mathrm{u}=\mathrm{v}=0.00003, \mathrm{u}=\mathrm{v}=0.000003$ successively. By programming, searching, and computing,... Until find the standard ASD model (AFER $=0 \%$, MSE $=0$ ). When $u=v=0.0003$, we can get table A, but AFER $\neq 0 \%$, MSE $\neq 0$; so continue to search, calculation, get the table $\mathrm{B}$, but $\mathrm{AFER} \neq 0 \%, \quad \mathrm{MSE} \neq 0$; continue to search, calculation, get the table $\mathrm{C}$, AFER $=0 \%$,MSE $=0$, terminate the calculation. So $A_{j}(0.000003,0.000003)$ is an ideal time series forecasting model in ASD when it predicts the number of 1971 1992 registrations at Alabama University.

TABLE I. USING $A_{J}(0.0003,0.0003)$ TO PREDICT THE NUMBER OF REGISTRATIONS AT ALABAMA UNIVERSITY

\begin{tabular}{ccccccc}
\hline Year & $\begin{array}{c}\text { Enrollments } \\
\mathrm{W}_{\mathrm{j}}\end{array}$ & $\begin{array}{c}\text { Difference } \\
\mathrm{Z}_{\mathrm{j}}\end{array}$ & $\begin{array}{c}\text { Forecast } \\
\mathrm{A}_{\mathrm{j}}\end{array}$ & $\mathrm{W}_{\mathrm{j}}-\mathrm{A}_{\mathrm{j}}$ & $\left(\mathrm{W}_{\mathrm{j}}-\mathrm{A}_{\mathrm{j}}\right)^{2}$ & $\mid \mathrm{W}_{\mathrm{j}}-\mathrm{A}_{\mathrm{j}} / \mathrm{W}_{\mathrm{j}}$ \\
\hline 1971 & 13055 & - & - & - & - & - \\
1972 & 13563 & 508 & 13563 & 0 & 0 & 0.000000 \\
1973 & 13867 & 304 & 13867 & 0 & 0 & 0.000000 \\
1974 & 14696 & 829 & 14696 & 0 & 0 & 0.000000 \\
1975 & 15460 & 764 & 15460 & 0 & 0 & 0.000000 \\
1976 & 15311 & -149 & 15311 & 0 & 0 & 0.000000 \\
1977 & 15603 & 292 & 15603 & 0 & 0 & 0.000000 \\
1978 & 15861 & 258 & 15861 & 0 & 0 & 0.000000 \\
1979 & 16807 & 946 & 16806 & 1 & 1 & 0.000059 \\
1980 & 16919 & 112 & 16919 & 0 & 0 & 0.000000 \\
1981 & 16388 & -531 & 16387 & 1 & 1 & 0.000061 \\
1982 & 15433 & -955 & 15433 & 0 & 0 & 0.000000 \\
1983 & 15497 & 64 & 15497 & 0 & 0 & 0.000000 \\
1984 & 15145 & -352 & 15144 & 1 & 1 & 0.000066 \\
1985 & 15163 & 18 & 15163 & 0 & 0 & 0.000000 \\
1986 & 15984 & 821 & 15973 & 11 & 121 & 0.000688 \\
1987 & 16859 & 875 & 16859 & 0 & 0 & 0.000000 \\
1988 & 18150 & 1291 & 18150 & 0 & 0 & 0.000000 \\
1989 & 18970 & 820 & 18970 & 0 & 0 & 0.000000 \\
1990 & 19328 & 358 & 19328 & 0 & 0 & 0.000000 \\
1991 & 19337 & 9 & 19337 & 0 & 0 & 0.000000 \\
1992 & 18876 & -461 & 18869 & 7 & 49 & 0.000371 \\
AFER & & & & & & $0.0059 \%$ \\
MSE & & & & & 8.2381 & \\
\hline
\end{tabular}


TABLE II.USING A $(0.00003,0.00003)$ TO PREDICT THE NUMBER OF REGISTRATIONS AT ALABAMA UNIVERSITY

\begin{tabular}{|c|c|c|c|c|c|c|}
\hline ear & $\begin{array}{l}\text { Enrollments } \\
\mathrm{W}_{\mathrm{j}}\end{array}$ & $\begin{array}{c}\text { Difference } \\
Z_{j} \\
\end{array}$ & $\begin{array}{l}\text { Forecast } \\
\mathrm{A}_{\mathrm{j}}\end{array}$ & $\mathrm{W}_{\mathrm{j}}-\mathrm{A}_{\mathrm{j}}$ & $\left(W_{j}-A_{j}\right)^{2}$ & $\left|\mathrm{~W}_{\mathrm{j}}-\mathrm{A}_{\mathrm{j}}\right| \mathrm{W}_{\mathrm{j}}$ \\
\hline 1971 & 13055 & - & - & - & - & - \\
\hline 1972 & 13563 & 508 & 13563 & 0 & 0 & 0.000000 \\
\hline 1973 & 13867 & 304 & 13867 & 0 & 0 & 0.000000 \\
\hline 1974 & 14696 & 829 & 14696 & 0 & 0 & 0.000000 \\
\hline 1975 & 15460 & 764 & 15460 & 0 & 0 & 0.000000 \\
\hline 1976 & 15311 & -149 & 15311 & 0 & 0 & 0.000000 \\
\hline 1977 & 15603 & 292 & 15603 & 0 & 0 & 0.000000 \\
\hline 1978 & 15861 & 258 & 15861 & 0 & 0 & 0.000000 \\
\hline 1979 & 16807 & 946 & 16807 & 0 & 0 & 0.000000 \\
\hline 1980 & 16919 & 112 & 16919 & 0 & 0 & 0.000000 \\
\hline 1981 & 16388 & -531 & 16388 & 0 & 0 & 0.000000 \\
\hline 1982 & 15433 & -955 & 15433 & 0 & 0 & 0.000000 \\
\hline 1983 & 15497 & 64 & 15497 & 0 & 0 & 0.000000 \\
\hline 1984 & 15145 & -352 & 15145 & 0 & 0 & 0.000000 \\
\hline 1985 & 15163 & 18 & 15163 & 0 & 0 & 0.000000 \\
\hline 1986 & 15984 & 821 & 15983 & 1 & 1 & 0.000063 \\
\hline 1987 & 16859 & 875 & 16859 & 0 & 0 & 0.000000 \\
\hline 1988 & 18150 & 1291 & 18150 & 0 & 0 & 0.000000 \\
\hline 1989 & 18970 & 820 & 18970 & 0 & 0 & 0.000000 \\
\hline 1990 & 19328 & 358 & 19328 & 0 & 0 & 0.000000 \\
\hline 1991 & 19337 & 9 & 19337 & 0 & 0 & 0.000000 \\
\hline 1992 & 18876 & -461 & 18875 & 1 & 1 & 0.000053 \\
\hline AFER & & & & & & $0.0006 \%$ \\
\hline MSE & & & & & 0.0952 & \\
\hline
\end{tabular}

TABLE III.

USING A $A_{J}(0.000003,0.000003)$ TO PREDICT THE NUMBER OF REGISTRATIONS AT ALABAMA UNIVERSITY

\begin{tabular}{ccccccc}
\hline Year & $\begin{array}{c}\text { Enrollments } \\
\mathrm{W}_{\mathrm{j}}\end{array}$ & $\begin{array}{c}\text { Difference } \\
\mathrm{Z}_{\mathrm{j}}\end{array}$ & $\begin{array}{c}\text { Forecast } \\
\mathrm{A}_{\mathrm{j}}\end{array}$ & $\mathrm{W}_{\mathrm{j}}-\mathrm{A}_{\mathrm{j}}$ & $\left(\mathrm{W}_{\mathrm{j}}-\mathrm{A}_{\mathrm{j}}\right)^{2}$ & $\mid \mathrm{W}_{\mathrm{j}}-\mathrm{A}_{\mathrm{j}} / \mathrm{W}_{\mathrm{j}}$ \\
\hline 1971 & 13055 & - & - & - & - & - \\
1972 & 13563 & 508 & 13563 & 0 & 0 & 0.000000 \\
1973 & 13867 & 304 & 13867 & 0 & 0 & 0.000000 \\
1974 & 14696 & 829 & 14696 & 0 & 0 & 0.000000 \\
1975 & 15460 & 764 & 15460 & 0 & 0 & 0.000000 \\
1976 & 15311 & -149 & 15311 & 0 & 0 & 0.000000 \\
1977 & 15603 & 292 & 15603 & 0 & 0 & 0.000000 \\
1978 & 15861 & 258 & 15861 & 0 & 0 & 0.000000 \\
1979 & 16807 & 946 & 16807 & 0 & 0 & 0.000000 \\
1980 & 16919 & 112 & 16919 & 0 & 0 & 0.000000 \\
1981 & 16388 & -531 & 16388 & 0 & 0 & 0.000000 \\
1982 & 15433 & -955 & 15433 & 0 & 0 & 0.000000 \\
1983 & 15497 & 64 & 15497 & 0 & 0 & 0.000000 \\
1984 & 15145 & -352 & 15145 & 0 & 0 & 0.000000 \\
1985 & 15163 & 18 & 15163 & 0 & 0 & 0.000000 \\
1986 & 15984 & 821 & 15984 & 0 & 0 & 0.000000 \\
1987 & 16859 & 875 & 16859 & 0 & 0 & 0.000000 \\
1988 & 18150 & 1291 & 18150 & 0 & 0 & 0.000000 \\
1989 & 18970 & 820 & 18970 & 0 & 0 & 0.000000 \\
1990 & 19328 & 358 & 19328 & 0 & 0 & 0.000000 \\
1991 & 19337 & 9 & 19337 & 0 & 0 & 0.000000 \\
1992 & 18876 & -461 & 18876 & 0 & 0 & 0.000000 \\
AFER & & & & & & $0.0000 \%$ \\
MSE & & & & & 0 & \\
\hline
\end{tabular}




\section{CONCLUSION}

For a time series, the application of computer can automatically search ideal prediction model in ASD, in which the MSE $=0, A F E R=0 \%$. and Table $C$ shows that the $A_{j}$ $(0.000003,0.000003)$ is the ideal prediction model when predict the registered number at Alabama University in year 1971 1992, which obtain the satisfactory prediction accuracy.

\section{ACKNOWLEDGMENT}

This work is supported by Natural Science Foundation of Hainan Province (Grant No.714283), and QYXB201301, 2016YD04, 2015YD33.

\section{REFERENCES}

[1] Q Song, B S Chissom. Forecasting enrollments with fuzzy time seriesPart I.Fuzzy Sets and Systems, Vol.54, pp. 1-9, 1993

[2] Q Song, B S Chissom. Fuzzy series and its models. Fuzzy Sets and Systems, Vol. 54, pp.269-277, 1993

[3] L A Zadeh. Fuzzy set .Fuzzy Sets and Systems, Vol. 8, pp. 338-353, 1965

[4] Tahseen A Jilani, S M Aqil Burney, and C Ardil. "Multivariate high order fuzzy time series Forecasting for car road accidents". World Academy of Science, Engineering and Technology, Vol. 1, pp. 288-293, 2007.

[5] T A Jilani, S M A Burney, C Ardil. Fuzzy metric approach for fuzzy time series forecasting based on frequency density based partitioning. Proceedings of World Academy of Science, Engineering and Technology, Vol.34, pp. 1-6, 2007.

[6] T A Jilani, S M A Burney. "M-factor high order fuzzy time series forecasting for road accident data”, IEEE-IFSA 2007, World Congress, Cancun, Mexico, June 18-21, Forthcoming in Book series Advances in Soft Computing, Springer-Verlag, 2007.

[7] C.H. Cheng, T.L. Chen, H.J. Teoh, C.H. Chiang, "Fuzzy time series based on adaptive expectation model for TAIEX forecasting (MEPA)", Expert Syst. Appl. vol. 34, pp.1126-1132, 2008.

[8] S.R. Singh, "A simple method of forecasting based on fuzzy time series”, Appl. Math. Comput, vol.186, pp. 330-339, 2007.

[9] V R Uslu, E Bas, U Yolcu, E Egrioglu. "A fuzzy time series approach based on weights determined by the number of recurrences of fuzzy relations", Swarm and Evolutionary Computation, vol.15, pp. 19-26, 2014.

[10] R C Tsaur, J C O Yang, H F Wang. "Fuzzy relation analysis in fuzzy time series model”, Comput. Math. Appl. vol. 49, pp. 539-548, 2005.

[11] C.H. Cheng, T.L. Chen, H.J. Teoh, C.H. Chiang, "Fuzzy time series based on adaptive expectation model for TAIEX forecasting (TFA)", Expert Syst. Appl. Vol.34, pp.1126-1132, 2008.

[12] T A Jilani, S M A Burney. "M-factor high order fuzzy time series forecasting for road accident data", IEEE-IFSA 2007, World Congress, Cancun, Mexico, June 18-21, Forthcoming in Book series Advances in Soft Computing, Springer-Verlag, 2007.

[13] C. Kai, F Fang-Ping, C Wen-Gang. "A novel forecasting model of fuzzy time series based on K-means clustering”, IWETCS, IEEE, pp. 223-225, 2010.

[14] T A Jilani, S M A Burney, C Ardil.“ Multivariate high order fuzzy time series forecasting for car road accidents", International Journal of Computational Intelligence, vol. 4, no. 1, pp. 15-20, 2007.

[15] Bhagawati P. Joshi and Sanjay Kumar., A computational method for fuzzy time series forecasting based on difference parameters. International Journal of Modeling, Simulation, and Scientific Computing, vol.4, no.1, pp. 1250023-1-1250023-12, 2013. 